\title{
microRNA-200a-3p increases 5-fluorouracil resistance by regulating dual specificity phosphatase 6 expression
}

\author{
Heejin Lee ${ }^{1}$, Chongtae Kim ${ }^{1}$, Hoin Kang ${ }^{1}$, Hyosun Tak ${ }^{1}$, Sojin Ahn ${ }^{1}$, Sungjoo Kim Yoon ${ }^{2,3}$, Hyo-Jeong Kuh ${ }^{2,3}$, \\ Wook Kim ${ }^{4}$ and Eun Kyung Lee ${ }^{1,3}$
}

Acquisition of resistance to anti-cancer drugs is a significant obstacle to effective cancer treatment. Although several efforts have been made to overcome drug resistance in cancer cells, the detailed mechanisms have not been fully elucidated. Here, we investigated whether microRNAs (miRNAs) function as pivotal regulators in the acquisition of anti-cancer drug resistance to 5-fluorouracil (5-FU). A survey using a lentivirus library containing 572 precursor miRNAs revealed that five miRNAs promoted cell survival after 5-FU treatment in human hepatocellular carcinoma Hep3B cells. Among the five different clones, the clone expressing miR-200a-3p (Hep3B-miR-200a-3p) was further characterized as a 5-FU-resistant cell line. The cell viability and growth rate of Hep3B-miR-200a-3p cells were higher than those of control cells after 5-FU treatment. Ectopic expression of a miR-200a-3p mimic increased, while inhibition of miR-200a-3p downregulated, cell viability in response to 5-FU, doxorubicin, and CDDP (cisplatin). We also showed that dual-specificity phosphatase 6 (DUSP6) is a novel target of miR-200a-3p and regulates resistance to 5-FU. Ectopic expression of DUSP6 mitigated the pro-survival effects of miR-200a-3p. Taken together, these results lead us to propose that miR-200a-3p enhances anti-cancer drug resistance by decreasing DUSP6 expression. Experimental \& Molecular Medicine (2017) 49, e327; doi:10.1038/emm.2017.33; published online 12 May 2017

\section{INTRODUCTION}

Multidrug resistance is a major factor leading to cancer treatment failure. It is a multifactorial phenomenon that includes the reduction of cell death, an increase in DNA repair, and alterations in drug metabolism. ${ }^{1-5}$ Because acquisition of drug resistance and toxic side effects limit the clinical applications of anti-cancer drugs, several studies have attempted to define the molecular and biochemical mechanisms related to the resistant phenotypes of cancer cells. ${ }^{6-10}$ However, the key determinants of drug resistance still remain largely unknown. 5-Fluorouracil (5-FU) is widely used to treat a range of cancers, including colorectal, liver, and breast cancers. ${ }^{7,11} 5$-FU is known to interfere with the synthesis of the pyrimidine thymidine, a nucleoside required for DNA replication, by inhibiting thymidylate synthase, thereby leading to cell cycle arrest or cell death in cancer cells. ${ }^{7}$ However, the acquisition of 5-FU resistance is one of the biggest obstacles for cancer therapy, and factors or mechanisms governing resistance need to be defined to increase the efficacy of chemotherapy.
microRNAs (miRNAs) are a conserved class of small non-coding RNAs that control gene expression by inducing mRNA degradation or by suppressing mRNA translation. ${ }^{12}$ miRNAs execute essential regulatory roles in cancer progression as oncogenes or tumor suppressors by affecting cell growth, death, migration, and differentiation; thus, their differential expression is linked to cancer development. ${ }^{13-16}$ In addition, several studies have highlighted emerging roles of miRNAs in anti-cancer drug resistance. ${ }^{5-9}$ Accumulating evidence suggests that the miR-200 family (miR-200a, -200b, $-200 c,-141$ and -429 ) regulates the pathogenesis of several types of cancer: it plays crucial roles in cell proliferation, metastasis, epithelial-mesenchymal transition, and anti-cancer drug resistance. ${ }^{17-24}$

Here, we demonstrated that miR-200a-3p is a novel factor regulating anti-cancer drug resistance in response to 5-FU. Using a lentivirus library containing miRNA precursors, we identified miR-200a-3p as a regulatory miRNA promoting cell survival after 5-FU treatment and investigated the correlation between miR-200a-3p and 5-FU resistance in human

\footnotetext{
${ }^{1}$ Department of Biochemistry, The Catholic University of Korea College of Medicine, Seoul, South Korea; ${ }^{2}$ Department of Medical Life Sciences, College of Medicine, The Catholic University of Korea, Seoul, South Korea; ${ }^{3}$ Cancer Evolution Research Center, The Catholic University of Korea College of Medicine, Seoul, South Korea and ${ }^{4}$ Department of Molecular Science and Technology, Ajou University, Suwon, South Korea

Correspondence: Professor EK Lee, Department of Biochemistry, The Catholic University of Korea, College of Medicine, 222 Banpodaero, Seocho-gu, Seoul 137-701, South Korea.

E-mail: leeek@catholic.ac.kr

Received 3 November 2016; revised 28 November 2016; accepted 5 December 2016
} 
hepatocellular carcinoma Hep3B cells. miR-200a-3p increased cell viability, while inhibition of miR-200a-3p sensitized Hep3B cells in response to 5-FU treatment. In silico analysis and a functional study further revealed that miR-200a-3p regulates the response to 5-FU in Hep3B cells by targeting dual-specific phosphatase 6 (DUSP6). Our study suggests that targeting the miR-200a-3p-DUSP6 axis could be a therapeutic strategy for overcoming chemoresistance in cancer therapy.

\section{MATERIALS AND METHODS}

\section{Establishment of 5-fluorouracil-resistant clones}

Human hepatocellular carcinoma Hep3B cells were transduced using a lentivirus library containing 572 precursor miRNAs (System Bioscience, Inc., Pala Alto, CA, USA) for $72 \mathrm{~h}$ (the multiplicity of infection was 10). To establish resistant cell lines, cells were re-plated in $100 \mathrm{~mm}$ dishes and incubated with medium containing $10 \mu \mathrm{m}$ 5-FU. Two weeks later, each colony was established as 5-FUresistant Hep3B clones.

\section{Identification of miRNAs in lentiviral clones}

To identify microRNAs overexpressed in lentivirus-infected clones, genomic DNA from each clone was prepared using an AccuPrep Genomic DNA extraction Kit (Bioneer Corp., Korea) according to the manufacturer's instructions, and the miRNA sequences integrated into genomic DNA were amplified by polymerase chain reaction with the specific primer pairs listed in Table 1. The amplicons containing miRNA sequences were isolated, and the sequences were determined using the sequencing primers pCDH-5.1-Fwd, 5'-GCCTGGAGA CGCCATCCACGCTG-3' and pCDH-3.1-Rvs, 5'-GATGTGCGCT CTGCCCACTGAC-3' (System Bioscience, Inc.).

\section{Cell culture, cloning and transfection}

Hep3B-CTRL, Hep3B-miR-200a and Hep3B cells were maintained with Dulbecco's modified Eagle's medium (Invitrogen, Carlsbad, CA, USA) containing $10 \%$ fetal bovine serum and $1 \%$ antibiotics. Enhanced green fluorescent protein (EGFP) reporter plasmids were constructed by insertion of the DUSP6 mRNA 3'UTR region (23852573, $189 \mathrm{nt}$ ) into pEGFP-C1 (BD Bioscience, San Jose, CA, USA). A mutant reporter lacking the binding sites for miR-200a-3p was generated by site-directed mutagenesis using a KOD-PlusMutagenesis Kit (Toyobo, Japan). The GFP-DUSP6 plasmid was a kind gift from Prof TH Kim at Chosun University. ${ }^{25}$ Transfection of plasmids or microRNA oligos was performed using Lipofectamine 2000 (Invitrogen).

Table 1 Primer sequences used for RT-PCR

\begin{tabular}{ll}
\hline Primer name & Sequence (5' to $\left.3^{\prime}\right)$ \\
\hline hsa-miR-200a-3p & TAACACTGTCTGGTAACGATGT \\
hsa-miR-200a-5p & CATCTTACCGGACAGTGCTGGA \\
pri-miR-200a-Fwd & TCACCGCTCCGGTTCTT \\
pri-miR-200a-Rvs & GCGGGTCACCTTTGAACATC \\
DUSP6-Fwd & GAAATGGCGATCAGCAAGACG \\
DUSP6-Rvs & CGACGACTCGTATAGCTCCTG \\
GAPDH-Fwd & TGCACCACCAACTGCTTAGC \\
GAPDH-Rvs & GGCATGGACTGTGGTCATGAG
\end{tabular}

Abbreviations: fwd, forward; miR, microRNA; RT-PCR, reverse transcriptase-PCR; rvs, reverse.

\section{MTT assay}

Cell viability was assessed using 3-(4,5-dimethylthiazol-2-yl)-2,5diphenyltetrazolium bromide (MTT). In brief, cells were transfected with miR-200a-3p mimic, inhibitor or control miRNA and incubated with anti-cancer drugs for $72 \mathrm{~h}$ and then further incubated with $0.5 \mathrm{mg} / \mathrm{ml}$ of MTT for $3 \mathrm{~h}$. Formazan crystals were solubilized with $100 \mu \mathrm{l}$ of $40 \mathrm{~mm}$ acidic isopropanol, and the absorbance was measured at $570 \mathrm{~nm}$ using a Victor 3 microplate reader (Perkin Elmer, Finland).

\section{RNA analysis and RT-qPCR}

Total RNAs were isolated using TRIzol reagent (Invitrogen). For mRNA analysis, cDNAs were prepared via reverse transcription using random hexamers and reverse transcriptase (Toyobo, Osaka, Japan). cDNAs for miRNA detection were synthesized using a Mir-X miRNA First Strand Synthesis kit (Clontech, Mountain View, CA, USA). The abundance of transcripts was assessed by quantitative PCR (qPCR) analysis using SYBR Green PCR master mix (Kapa Biosystems, Wilmington, MA, USA) and gene-specific primer sets on a StepOne Plus instrument (Applied Biosystems, Foster City, CA, USA). GAPDH mRNA and U6 RNA were used as the internal controls for normalization. Primer sequences are listed in Table 1.

\section{Flow cytometric analysis}

Cell cycle distribution was assessed using fluorescence-activated cellsorting (FACS) analysis by tracing propidium iodide-stained nuclei as previously described. ${ }^{26}$ After 5-FU treatment, cells were incubated with propidium iodide (Sigma Aldrich, St Louis, MO, USA) and analyzed by flow cytometry using a FACSCalibur flow cytometer (BD Biosciences).

\section{Western blotting}

Whole cell lysates were prepared using RIPA buffer (10 mm Tris- $\mathrm{HCl}$ ( $\mathrm{pH}$ 7.4), $150 \mathrm{~mm} \mathrm{NaCl}, 1 \% \mathrm{NP}-40,1 \mathrm{~mm}$ EDTA, and 0.1\% SDS), separated by electrophoresis in SDS-containing polyacrylamide gels, and transferred onto PVDF membranes (EMD Millipore, Billerica, MA, USA). The membranes were incubated with primary antibodies against DUSP6 and EGFP (Santa Cruz Biotechnology, Santa Cruz, CA, USA) or $\beta$-actin (Abcam, Cambridge, MA, USA) and then further incubated with the appropriate secondary antibodies conjugated to horseradish peroxidase (Santa Cruz Biotechnology). Chemiluminescent signals were visualized using NEW Clarity ECL substrate (Bio-Rad, Hercules, CA, USA). For quantitative analysis, the density of the individual images was analyzed using Image J software $(\mathrm{NIH}$, Bethesda, MD, USA).

\section{Colony forming assay}

After incubating cells with 5 -FU or DMSO for $72 \mathrm{~h}, 1 \times 10^{3}$ cells per well were cultured for 2 weeks. Cells were fixed with $4 \%$ paraformaldehyde and stained with $0.05 \%$ crystal violet for $10 \mathrm{~min}$ at room temperature. After washing and drying the plates, the number of colonies was estimated by counting colonies from three random fields $\left(35 \mathrm{~mm}^{2}\right)$ per sample.

\section{RESULTS}

\section{Establishment of resistant Hep3B-Lenti-miR clones to} 05-fluorouracil

To identify miRNAs involved in the acquisition of anti-cancer drug resistance, we transduced lentiviral particles containing 572 precursor miRNAs into human hepatocellular carcinoma 
a

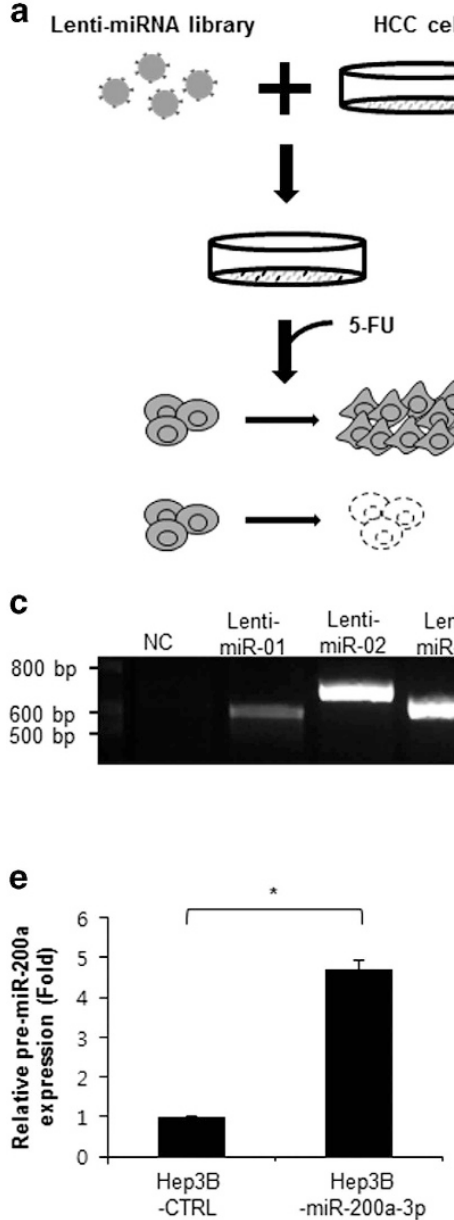

b

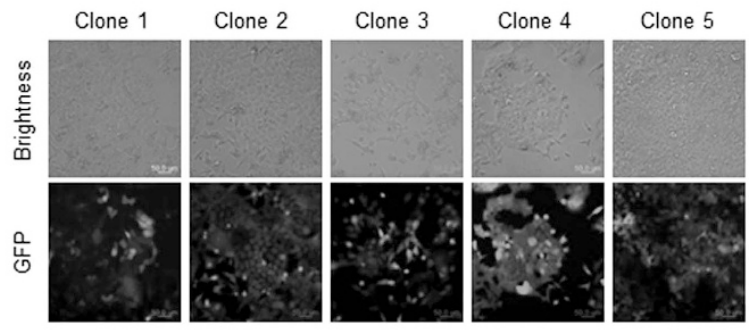

d

\begin{tabular}{ccc}
\hline Clones & Sequence matched miRNAs & Score \\
\hline Lenti-miR-01 & hsa-miR-602 & 319 \\
Lenti-miR-02 & hsa-miR-200a & 423 \\
Lenti-miR-03 & hsa-miR-551a & 188 \\
Lenti-miR-04 & hsa-miR-1322 & 93 \\
Lenti-miR-05 & hsa-miR-561 & 88 \\
\hline
\end{tabular}

miR-amplicons
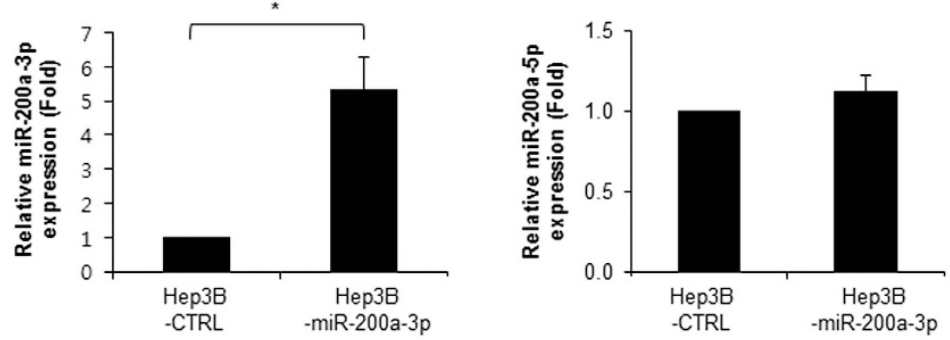

Figure 1 5-FU-resistant Hep3B clones were established using a lentivirus library. (a) Hep3B cells were transduced with a lentivirus library containing 572 miRNAs (multiplicity of Infection, $\mathrm{MOI}=10$ ) and incubated with $10 \mu \mathrm{m}$ 5-FU until control cells were dead. (b) Five of each of the surviving clones were isolated and established as 5-FU-resistant clones. (c, d) miR-amplicons from each resistant clone was prepared by genomic DNA PCR using specific primer sets, and the sequences were analyzed as described in the Materials and Methods section. (e) Relative levels of precursor miR-200a, miR-200a-3p, and miR-200a-5p were assessed by RT-qPCR. GAPDH mRNA and U6 RNA were used as internal controls. The results represent the mean \pm s.e.m. from three independent experiments. ${ }^{*} P<0.05$. miRNA, microRNA; RT-qPCR, real-time quantitative PCR; 5-FU, 5-flurouracil.

Hep3B cells, with a multiplicity of infection of 10 , and incubated the cells with $10 \mu \mathrm{M}$ 5-FU until the transduced cells expressing control miRNA (Hep3B-CTRL) were dead (Figure 1a). Each Hep3B clone that survived after the transduction of lenti-miRNA particles and incubation with 5-FU was isolated and established as a 5-FU-resistant clone (Figure 1b). Individual miRNAs from each resistant clone were identified by genomic DNA PCR using specific primer sets followed by sequencing of PCR amplicons (Figure 1c). Five microRNAs were identified from the 5 different 5-FU-resistant Hep3B clones listed in Figure 1d. Among them, we further characterized the Lenti-miR-02 clone expressing miR-200a. Further qPCR analysis using specific primer sets revealed that the relative levels of pre-miR-200a and miR-200a-3p, but not miR-200a-5p, were higher in the Hep3B-Lenti-miR-02 clone compared to Hep3B-CTRL cells (Figure 1e). These results indicate that miR-200a-3p has a potential role in the acquisition of 5-FU resistance in Hep3B cells.
miR-200a-3p decreased 5-FU sensitivity in Hep3B cells.

Because we identified miR-200a-3p in the surviving Hep3B clones after 5-FU treatment, we investigated the 5-FU sensitivity of both Hep3B-miR-200a-3p and Hep3B-CTRL cells with an MTT assay. As shown in Figure 2a, 5-FU treatment resulted in a dose-dependent reduction in cell viability in both Hep3B clones. However, we observed that Hep3B-miR-200a-3p cells were more resistant to 5-FU than Hep3B-CTRL cells (Figure 2a). We also analyzed the colony-forming ability of each clone after 5-FU treatment and observed an increase in colony numbers in the Hep3B-miR-200a-3p clone (Figure 2b), which indicates that higher expression of miR-200a-3p is related to an increase in cell viability as well as growth in response to 5-FU. To further characterize Hep3B-miR-200a-3p clones after treatment with 5-FU, the cell cycle distribution of each clone was investigated with a FACS analysis, and the population of cells in the subG1 phase was determined (Figure 2c). The subG1 population was decreased after 

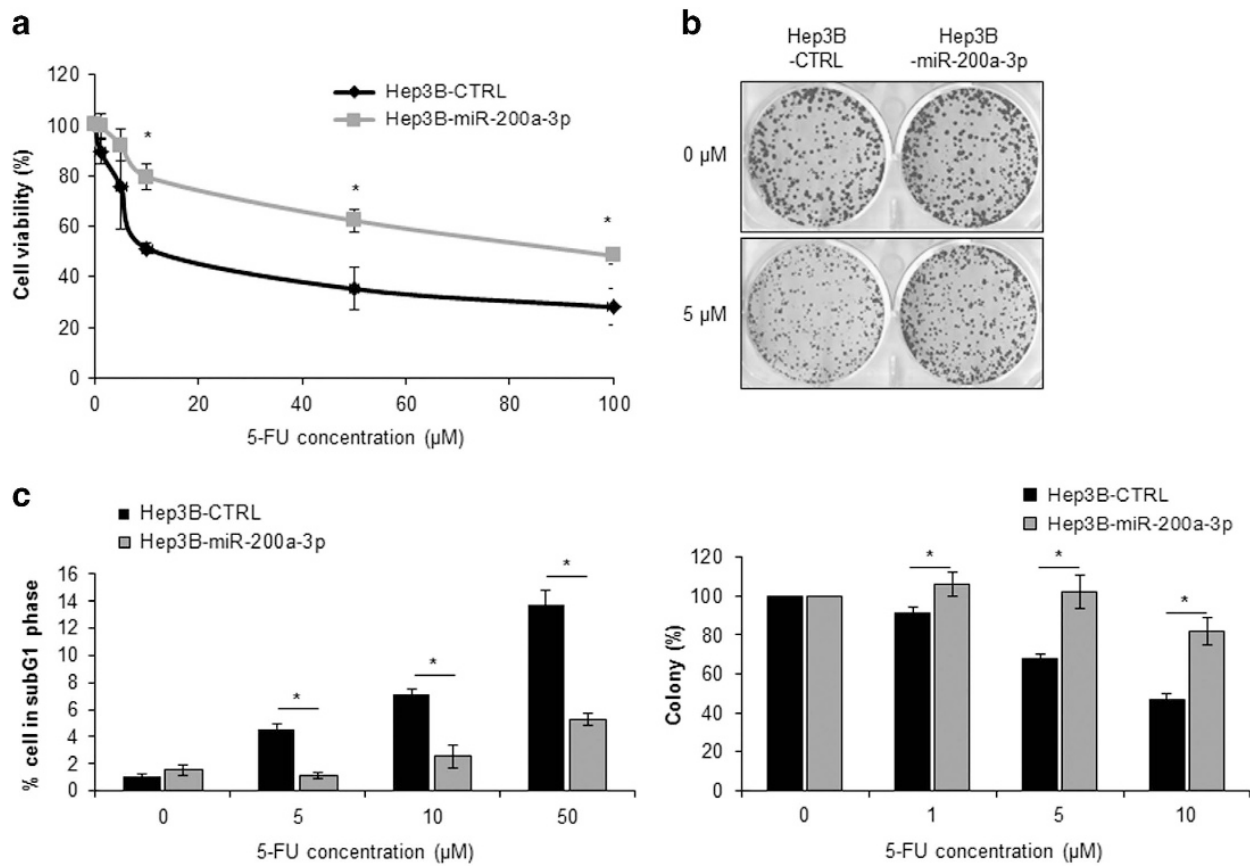

Figure 2 Hep3B clones expressing miR-200a-3p have reduced chemosensitivity to 5-FU. (a) Hep3B clones expressing either miR-200a-3p or control plasmid were incubated with 5-FU for $72 \mathrm{~h}$, and cell viability was assessed with an MTT assay. (b) To analyze the colonyforming activity, $1 \times 10^{3}$ cells of each clone were cultured for 2 weeks, and the colonies were stained with crystal violet. The number of colonies in three independent experiments was analyzed. (c) Both clones were incubated with 5-FU for $48 \mathrm{~h}$ and stained with propidium iodide, and then, the cell cycle distribution was analyzed by flow cytometry. The relative portion of the subG1 phase from either Hep3BCTRL or Hep3B-miR-200a-3p cells was analyzed. The results represent the mean \pm s.e.m. or are representative of 3 independent experiments. ${ }^{*} P<0.05$. miR, microRNA; 5-FU, 5-flurouracil.

5-FU treatment in the Hep3 B-miR-200a-3p clone. From these results, we speculated that the Hep3B-miR-200a-3p clones showed enhanced resistance against 5-FU and that miR-200a-3p had the potential to regulate the sensitivity to 5-FU. To further test this hypothesis, we ectopically transfected miR-200a-3p mimic or anti-miR-200a-3p into Hep3B cells and analyzed the cell viability after incubating cells with 5 -FU for $72 \mathrm{~h}$. We observed that ectopic expression of miR-200a-3p increased the cell viability, while inhibition of miR-200a-3p decreased it, after 5-FU treatment (Figure 3a). We also analyzed the colony-forming ability after 5-FU treatment in miR-200a-3p mimic or anti-miR-200a-3p transfected cells. Ectopic expression of miR-200a-3p mimic promoted colony formation in Hep3B cells after 5-FU treatment; however, inhibition of miR-200a-3p resulted in a reduction in colony formation (Figure 3b). In addition, miR-200a-3p inhibition decreased the viability of Hep3B-miR-200a-3p clones in response to $5-\mathrm{FU}$ (Figure $3 \mathrm{c}$ ). These results indicate that a high level of miR-200a-3p is responsible for the survival of Hep3B cells in response to 5-FU and that inhibition of miR-200a-3p has the potential to sensitize Hep3B cells, thereby leading to cell death after 5-FU treatment.

\section{miR-200a-3p desensitized Hep3B cells to CDDP and doxorubicin}

$5-\mathrm{FU}$ is an anti-cancer drug that triggers cell death by inhibiting thymidine synthase. ${ }^{7}$ To evaluate whether
miR-200a-3p regulates anti-cancer drug resistance via 5-FU, we investigated the regulation of cell viability by miR-200a-3p after treatment with two different types of anti-cancer drugs, CDDP and doxorubicin. As shown in Figures $4 \mathrm{a}$ and $\mathrm{b}$, transfection of the miR-200a mimic into Hep3B cells increased cell viability after CDDP and doxorubicin treatment. However, inhibition of miR-200a-3p sensitized Hep3B cells to CDDP and doxorubicin, leading to cell death (Figure 4a). In addition, we further evaluated the effect of miR-200a-3p inhibition in resistant Hep3B clones and observed that miR-200a-3p inhibition decreased cell viability in both Hep3B-CTRL and Hep3BmiR-200a-3p clones in response to CDDP and doxorubicin (Figure $4 \mathrm{~b}$ ). We also investigated the pro-survival effect of miR-200a in response to anti-cancer drugs using two different cell lines, including human colorectal carcinoma HCT116 and human hepatic adenocarcinoma SK-Hep1 cells, and observed that miR-200a expression resulted in an increase in cell viability after 5-FU, CDDP, and doxorubicin treatment in both cell lines (Supplementary Figure S1) These results suggest that miR-200a-3p is involved in the promotion of cell survival in response to various anti-cancer drugs and not specific only to 5 -FU.

\section{miR-200a-3p decreased DUSP6 expression by binding its} $3^{\prime}$ UTR

To identify the target mRNAs of miR-200a-3p, we performed in silico analysis using three different miRNA prediction 
a
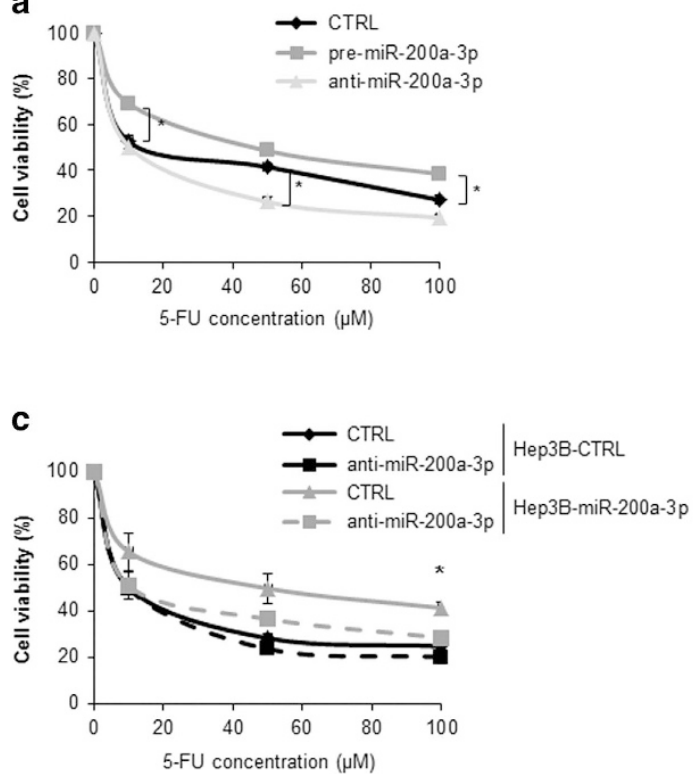

b
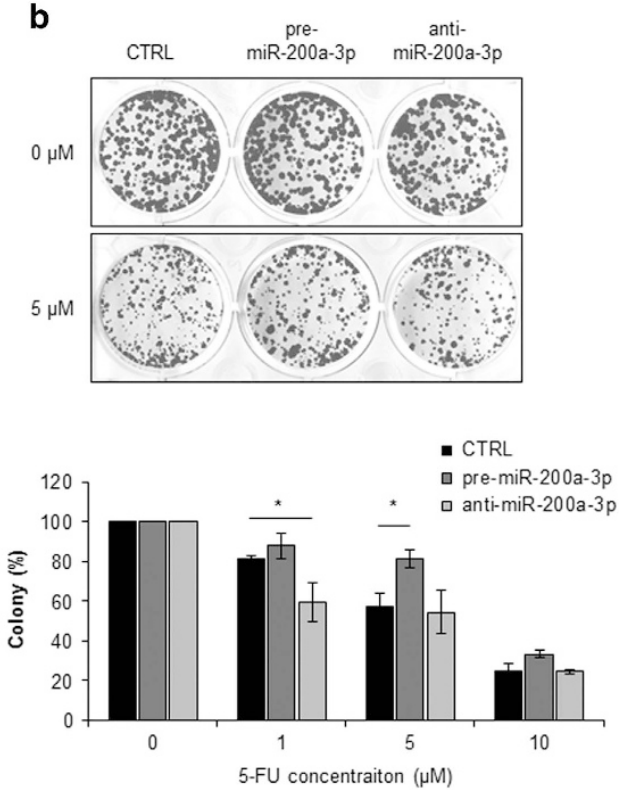

Figure 3 miR-200a-3p desensitizes Hep3B cells to 5-FU. Hep3B cells were transfected with control miRNA (CTRL), miR-200a-3p mimic (pre-miR-200a) or inhibitor of miR-200a-3p (anti-miR-200a-3p) for $48 \mathrm{~h}$ and further incubated with 5 -FU for $72 \mathrm{~h}$. Cell viability and growth were assessed with an MTT assay (a) or colony formation assay (b), respectively. (c) Both Hep3B-CTRL and Hep3B-miR-200a clones were transfected with anti-miR-200a-3p. After incubating cells with 5-FU for $72 \mathrm{~h}$, cell viability was assayed with an MTT assay. The results represent the mean \pm s.e.m. or are representative of 3 independent experiments. ${ }^{*} P<0.05$. miR, microRNA; 5-FU, 5-flurouracil.
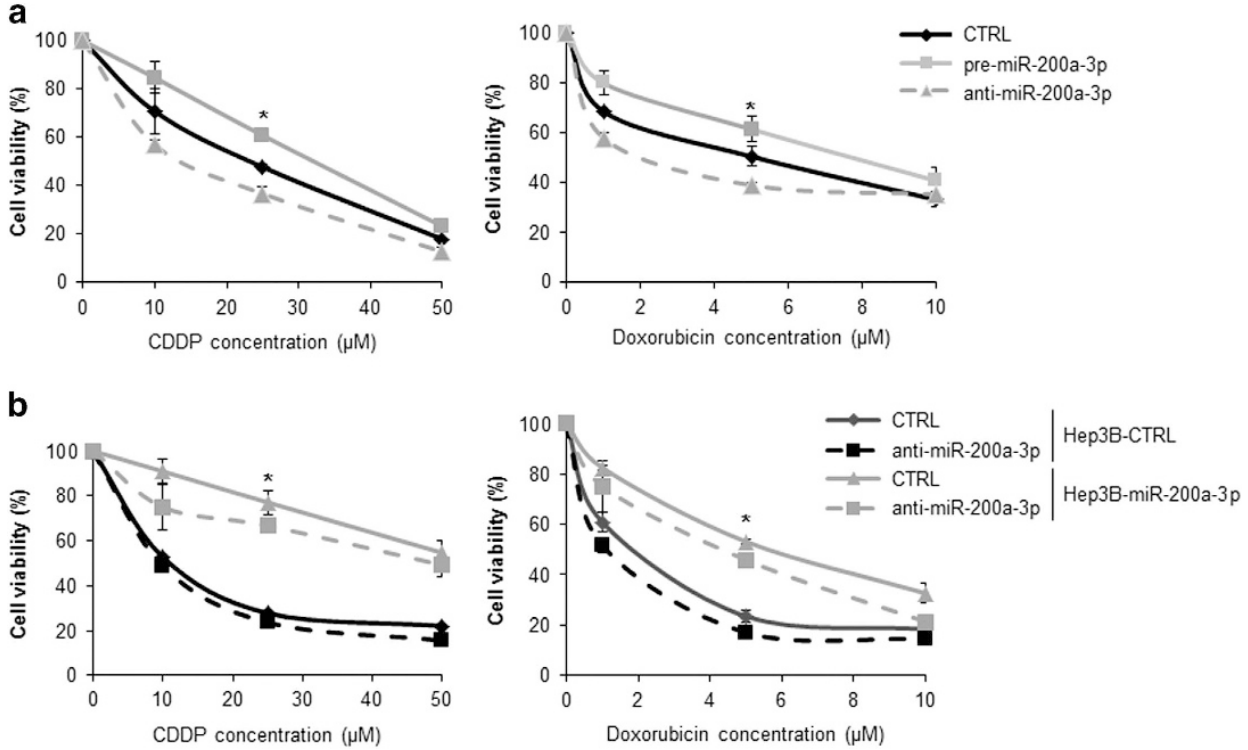

Figure 4 miR-200a-3p desensitizes Hep3B cells to CDDP and doxorubicin. (a) Hep3B cells were transfected with control miRNA (CTRL), miR-200a-3p mimic (pre-miR-200a-3p) or inhibitor of miR-200a-3p (anti-miR-200a-3p) for $48 \mathrm{~h}$ and further incubated with CDDP (left) or doxorubicin (right) for $72 \mathrm{~h}$. (b) Both Hep3B-CTRL and Hep3B-miR-200a-3p clones were transfected with anti-miR-200a and further incubated with CDDP (left) or doxorubicin (right) for $72 \mathrm{~h}$. Cell viability after treatment with anti-cancer drugs was assessed with an MTT assay. The results represent the mean \pm s.e.m. from 3 independent experiments. ${ }^{\star} P<0.05$. miR, microRNA; 5-FU, 5-flurouracil.

algorithms (Targetscan, miRWalk, and miRDB) and the PANTHER classification system. ${ }^{27-31}$ A survey revealed that five mRNAs, dual specificity phosphatase 6 (DUSP6), Insulinlike growth factor 2 mRNA-binding protein 2 (IGF2BP2), Poly (rC)-binding protein 3 (PCBP3), Unc-51-like kinase 2 (ULK2) and tumor necrosis factor receptor superfamily member $10 \mathrm{~b}$ (TNFRSF10B) are putative targets of miR-200a-3p (Figure 5a). Among these targets, we examined DUSP6 regulation by miR-200a-3p. To assess the roles of miR-200a-3p in DUSP6 regulation, we analyzed both mRNA and protein levels after 
a

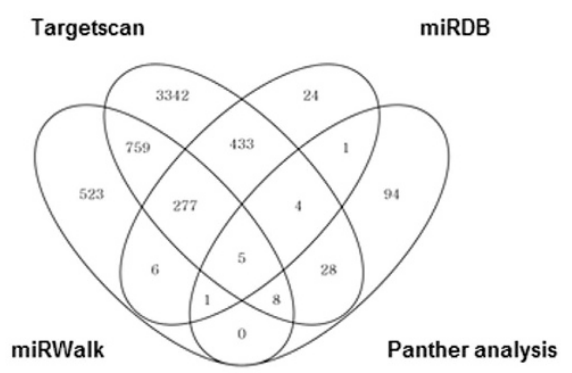

b
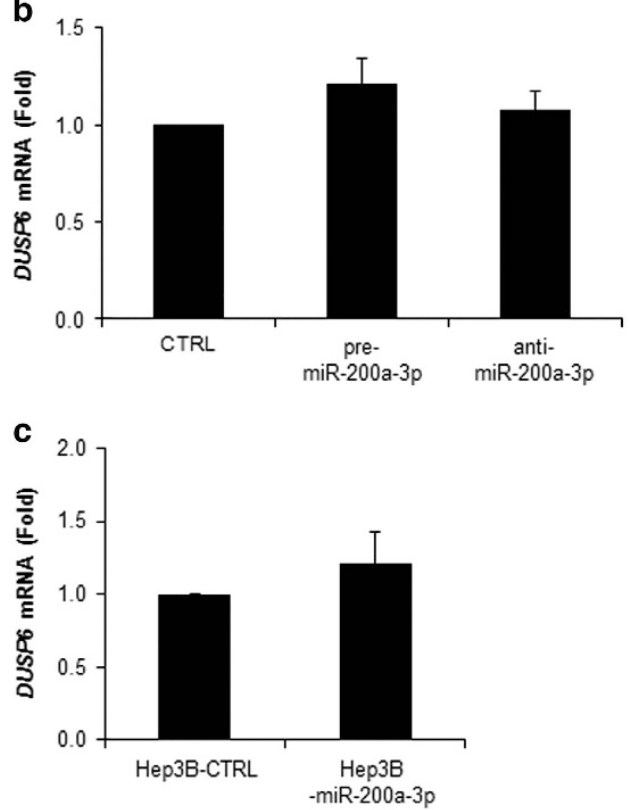

d

pEGFP
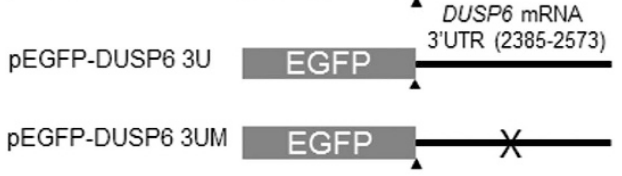

5'...cuugaaUCACUUGACAgUGUUu...3' DUSP6 mRNA

| ||: |||||||||

3' ...uguagcAAUGGUCUGUCACAAu...5' miR-200a-3p

| ||: || ||

5'...cuugaaUCACUUCUGUCACAAu...3' Mutant DUSP6 mRNA
DUSP6

IGF2BP2

$\mathrm{PCBP} 3$

ULK2

TNFRSF10B

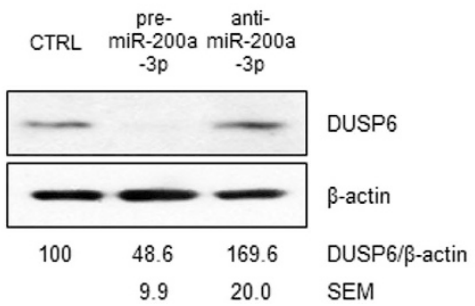

Hep3B Hep3B

-CTRL -miR-200a-3p

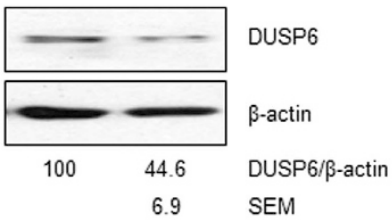

e

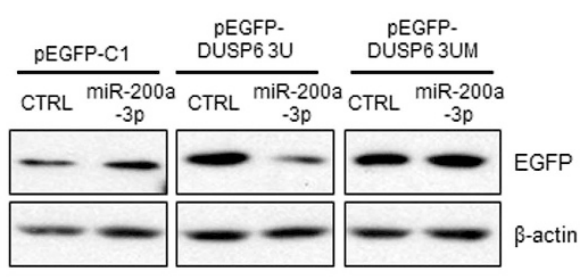

Figure 5 miR-200a-3p decreases DUSP6 expression by binding to its 3'UTR. (a) In silico analysis of putative target mRNAs of miR-200a3p. mRNAs predicted as targets of miR-200a-3p from three different algorithms (Targetscan, miRDB, and miRWalk) and involved in apoptosis/cell growth (Panther Classification System) were analyzed, and the results are shown as a simple list. (b, c) After transfection with CTRL miRNA, pre-miR-200a-3p, and anti-miR-200a-3p for $48 \mathrm{~h}$, DUSP6 mRNA and DUSP6 protein levels were assessed by RT-qPCR (left) and western blot analysis (right) in miRNA-transfected cells and resistant clones. GAPDH mRNA and $\beta$-actin were used for normalization and as a loading control, respectively. (d) A schematic of the EGFP-reporter constructs. The 3'UTR of DUSP6 mRNA containing a miR-binding site was inserted into pEGFP-C1 (pEGFP-DUSP6 3U); a mutant reporter construct lacking the miR-binding site was generated using site-directed mutagenesis (pEGFP-DUSP6 3UM). (e) Reporter analysis after miR-200a-3p expression. After transfection of the cells with miR-200a-3p and reporter constructs along with a control vector, EGFP levels were assessed by western blotting. $\beta$-actin served as a loading control. Bar or number indicate the mean \pm s.e.m., and the image is representative of three independent experiments. miR, microRNA; RT-qPCR, real-time quantitative PCR; 5-FU, 5-flurouracil.

transfection with miR-200a-3p mimic, anti-miR-200a-3p, and control miRNA. There were no significant changes in the DUSP6 mRNA level after miRNA transfection; however, the
DUSP6 protein levels were negatively downregulated by miR-200a-3p (Figure 5b). We also investigated the DUSP6 levels in the Hep3B-miR-200a-3p clone and confirmed that the 

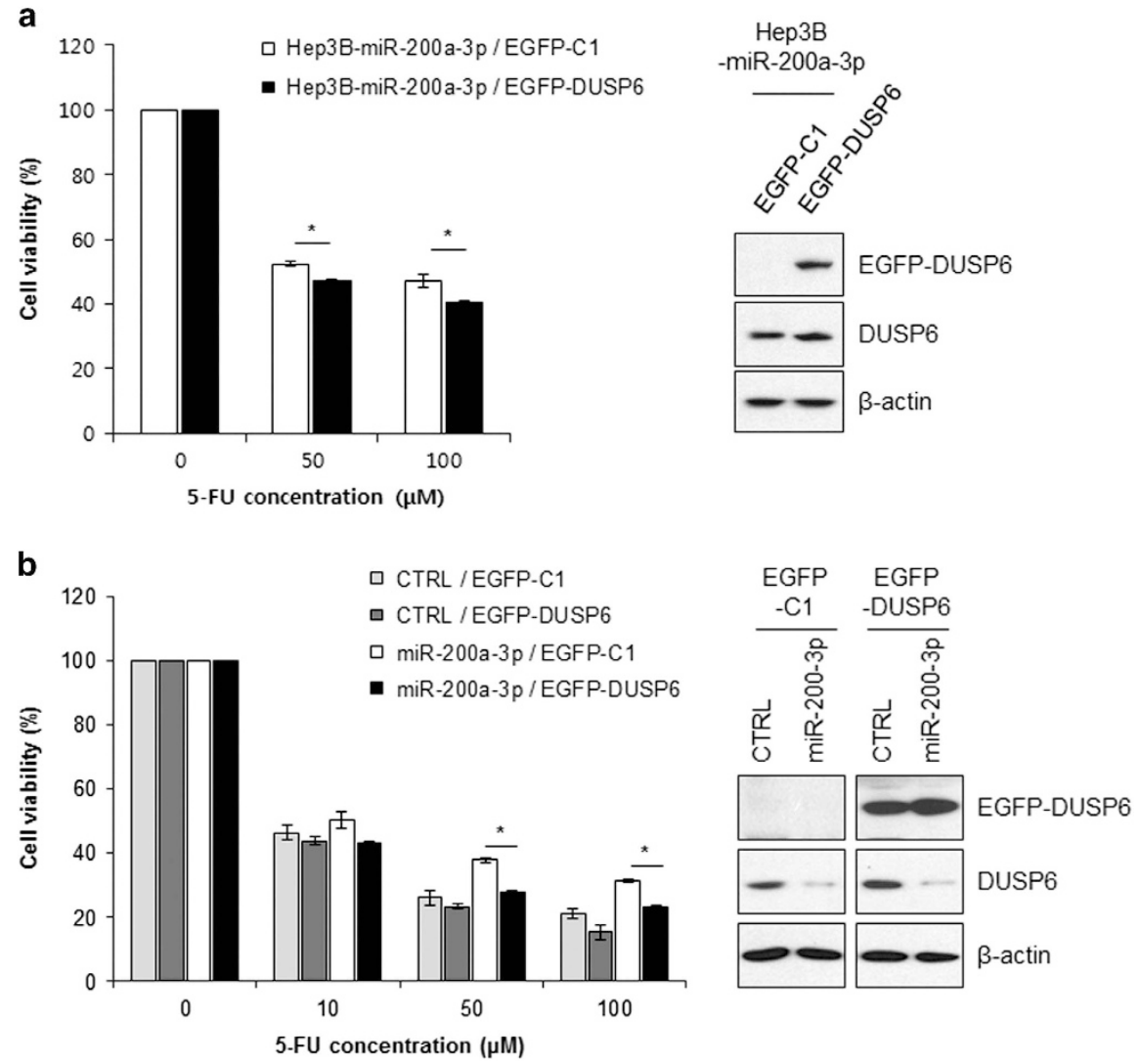

Figure 6 Ectopic expression of DUSP6 alleviates the pro-survival effect of miR-200a-3p in response to 5-FU. (a) A Hep3B-miR-200a-3p clone was transfected with pEGFP-DUSP6 or pEGFP control plasmids and further incubated with 5-FU for $72 \mathrm{~h}$. (b) After transfection with miR-200a-3p mimic or control miRNA, Hep3B cells were further transfected with pEGFP-DUSP6 or pEGFP and treated with 5-FU for $72 \mathrm{~h}$. Cell viability and DUSP6 levels were assessed with an MTT assay (left) and western blotting (right), respectively. Bar indicates the mean \pm s.e.m., and the image is representative of three independent experiments. ${ }^{*} P<0.05$. miR, microRNA; 5-FU, 5-flurouracil.

Hep3B-miR-200a-3p clone had a lower expression of DUSP6 without significant changes in its mRNA level (Figure 5c). To further examine DUSP6 regulation by miR-200a-3p, we generated EGFP reporter constructs containing the DUSP6 mRNA 3'UTR (2385-2573 nt) (pEGFP-DUSP6 3U) or mutant $3^{\prime}$ UTR missing the seed region binding sites for miR-200a-3p (pEGFP-DUSP6 3UM) (Figure 5d), and then, analyzed EGFP reporter expression after miR-200a-3p overexpression. As shown in Figure 5e, miR-200a-3p downregulated EGFPDUSP6 3U reporter expression but did not affect the EGFP control or EGFP-DUSP6 3UM. These results indicate that DUSP6 is the target of miR-200a-3p.

\section{miR-200a-3p de-sensitized cells to 5 -FU by downregulating} DUSP6 expression

We observed that miR-200a-3p increased cell viability after 5-FU treatment (Figure 3) and downregulated DUPS6 expression (Figure 5). To further determine whether the increase in $5-\mathrm{FU}$ resistance by miR-200a-3p is mediated by the downregulation of DUSP6, we investigated the sensitivity of Hep3BmiR-200a-3p cells to 5-FU after ectopic expression of a DUSP6 construct containing only the coding sequence. ${ }^{25}$ DUSP6 overexpression decreased cell viability after 5 -FU treatment in
Hep3B-miR-200a-3p cells, which indicates that DUSP6 sensitized Hep3B-miR-200a-3p cells (Figure 6a). In addition, we analyzed cell viability after DUSP6 overexpression in miR-200a-3p transfected cells. As shown in Figure 6b, increased viability by miR-200a-3p was downregulated by ectopic expression of DUSP6, which indicates that DUSP6 alleviates the pro-survival effects of miR-200a-3p in response to 5-FU. These results suggest that the miR-200a-3p-DUSP6 axis has a novel role in the regulation of the 5-FU response in Hep3B cells.

\section{DISCUSSION}

Multidrug resistance is one of the major obstacles for chemotherapy. Understanding the mechanisms of both intrinsic and acquired resistance is important for providing specific targeted therapy and to gain novel insights into the regulation of cancer progression, as well as for the development of novel agents. ${ }^{1,32}$ Although several efforts have been made to overcome multidrug resistance, the mechanism of drug resistance still remains largely unknown. In this study, we investigated the underlying mechanisms of miRNAs in the acquisition of drug resistance. To identify miRNAs as active drivers that regulate the acquisition of 5-FU resistance, we 
established 5-FU-resistant Hep3B cell lines after transducing lentivirus particles containing 572 miRNAs. Five GFP-positive and resistant clones were established after sequential exposure to 5-FU, and individual miRNAs (miR-602, miR-200a, miR-551a, miR-1322 and miR-561) were identified, as shown in Figure 1. After another round of validation, we further investigated the role of miR-200a-3p in the acquisition of 5 -FU resistance in $\mathrm{Hep} 3 \mathrm{~B}$ cells. We demonstrated that miR-200a-3p promoted cell survival after 5-FU treatment and that the inhibition of miR-200a-3p sensitized Hep3B cells to 5-FU. Additionally, we showed that miR-200a-3p increased cell survival in response to CDDP and doxorubicin, which indicates that miR-200a-3p mediates resistance to cancer drugs, including 5-FU, CDDP and doxorubicin. We further showed that miR-200a-3p regulated cell survival via DUSP6 downregulation in response to $5-\mathrm{FU}$.

The miR-200 family consists of 5 different miRNAs (miR-200a, -b, -c, -141 and miR-429) and plays multiple roles by regulating cancer initiation and metastasis in various cancers. ${ }^{17,19,20,23}$ It was previously reported that miR-200s have tumor suppressive effects on cell transformation, proliferation, migration, and invasion by downregulating various target mRNAs such as the E-cadherin repressors ZEB1/2 (SIP1), and in particular, miR-200b/c functions as a tumor suppressor in certain cell types. ${ }^{19-21,33}$ In addition, previous studies have shown that miR-200a is involved in breast cancer development by regulating specific target mRNAs, including YAP1, KEAP1, EPHA2 and SIRT1. ${ }^{23,34-37}$ It was also reported that miR-200a regulates the expression of MAPK14, STAT5B, SIM2-s and $\beta$-catenin in ovarian, kidney and brain cancer. ${ }^{38-40}$ Here, we demonstrated that miR-200a-3p promoted the acquisition of resistance to 5-FU by targeting DUSP6, thereby promoting cell survival after 5-FU treatment. Minimal evidence makes it difficult to determine the dominant function of the miR-200 family as enhancers or suppressors of cancer progression. It seems that the controversial roles of the miR-200 family may be due to its relative level and differential regulation in certain cell or cancer types. Further functional validation is needed to understand the roles of the miR-200 family in various types of cancer.

DUSP6/MKP-3 is a cytoplasmic, ERK-specific phosphatase that plays a crucial role in cancer development and progression by dephosphorylating ERK1/2. ${ }^{41,42}$ It has been reported that DUSP6 is upregulated in myeloma, melanoma, glioma, breast and pancreatic cancer, whereas it is downregulated in pancreatic ductal carcinoma, lung and ovarian cancer. ${ }^{43-51}$ While DUSPs/MKPs have a crucial role in cancer progression, how they are differentially expressed in certain cell types or certain circumstances is not fully understood. It has been reported that expression of DUSPs/MKPs could be modulated by p53, FGF or $\beta$-catenin signaling ${ }^{25,52,53}$ and that their activity is differentially regulated by post-translational modifications, such as phosphorylation or ubiquitination. ${ }^{54,55}$ In addition, several factors affecting DUSP expression at the RNA level have been identified. For example, the RNA-binding proteins HuR and NF90 are involved in the regulation of DUSP1 expression by stabilizing DUSP1 mRNA. ${ }^{56}$ Additionally, miR-29b downregulated DUSP2 expression, and miR-145/181a/125b were involved in a decrease in DUSP6 levels. ${ }^{57-60}$ In this study, we provided experimental evidence that miR-200a-3p is a novel regulator that reduces DUSP6 expression (Figure 5).

Because DUSPs/MKPs play a role in both cancer progression and cancer resistance, ${ }^{25}$ targeting DUSPs/MKPs could provide novel therapeutics. In this study, we demonstrated that a high level of miR-200a-3p is responsible for an increase in resistance to 5 -FU (Figure 2), and inhibition of miR-200a-3p decreased cell growth and viability after 5-FU treatment, thereby sensitizing Hep3B cells to anti-cancer drugs, including 5-FU, CDDP, and doxorubicin (Figures 3 and 4). These results suggest that inhibition of miR-200a-3p has the potential to reduce chemoresistance in response to various anti-cancer drugs. The relative expression levels of miR-200a-3p among 5-FU-resistant cells or in patient groups with chemoresistance were not assessed in this study. Further analysis of the differential expression of miR-200a-3p in various types of resistant cells would be helpful in understanding the relationship between drug resistance and miR-200a-3p. Previous studies reported that miR-200a inhibited doxorubicin-induced cell death, ${ }^{61}$ and a high level of miR-200a in hepatocellular carcinoma cells increased resistance to curcumin. ${ }^{62}$ To assess whether 5-FU affects miR-200a-3p expression, we investigated the levels of miR-200a-3p and pre-miR-200a after 5-FU treatment in Hep3B cells. There were no significant changes in miR-200a$3 \mathrm{p}$ level until $72 \mathrm{~h}$ after 5 -FU treatment (data not shown). Because the acquisition of drug resistance may not occur within a short period, another approach is needed to determine whether 5-FU directly regulates miR-200a-3p expression and whether the differential level of miR-200a-3p is a driving force leading to the acquisition of drug resistance. In addition, the clinical significance of miR-200a-3p in chemoresistance requires further investigation. In this study, we demonstrated that stable expression of miR-200a-3p promoted cell survival in response to anti-cancer drugs, and miRNA inhibition using anti-miR-200a-3p sensitized cells via DUSP6 regulation. These results indicate that miR-200a-3p plays an important role in promoting cell survival in response to 5-FU, and the miR-200a-3p-DUSP6 axis is involved in the acquisition of drug resistance. Our study provides novel insights into the function of miR-200a-3p, which enable the development of a prognostic marker or therapeutic strategy using antimiR-200a-3p to increase the efficacy of chemotherapy.

\section{CONFLICT OF INTEREST}

The authors declare no conflict of interest.

\section{ACKNOWLEDGEMENTS}

We would like to thank Professor TH Kim at Chosun University for providing the EGFP-DUSP6 plasmid. This work is supported by a National Research Foundation of Korea (NRF) grant funded by the Korea government (2014R1A2A1A11053431, 2012M3A9D1054517, 2012R1A5A2047939, and 2009-0093826). 
Author contributions: HL and EKL designed the experiments and wrote the manuscript. CK established stable cell lines. HL, CK, HK, HT and SA performed experiments. HL, WK and EKL revised the manuscript, and all authors reviewed the manuscript.

1 Gottesman MM. Mechanisms of cancer drug resistance. Annu Rev Med 2002; 53: 615-627.

2 Gottesman MM, Lavi O, Hall MD, Gillet JP. Toward a Better Understanding of the Complexity of Cancer Drug Resistance. Annu Rev Pharmacol Toxicol 2016; 56: 85-102.

3 Ma J, Dong C, Ji C. microRNA and drug resistance. Cancer Gene Ther 2010; 17: 523-531.

4 Zheng T, Wang J, Chen X, Liu L. Role of microRNA in anticancer drug resistance. Int J Cancer 2010; 126: 2-10.

5 Naidu S, Garofalo M. microRNAs: An Emerging Paradigm in Lung Cancer Chemoresistance. Front Med (Lausanne) 2015; 2: 77.

6 Magee P, Shi L, Garofalo M. Role of microRNAs in chemoresistance. Ann Trans/ Med 2015; 3: 332.

7 Longley DB, Harkin DP, Johnston PG. 5-fluorouracil: mechanisms of action and clinical strategies. Nat Rev Cancer 2003; 3: 330-338.

8 Riquelme I, Letelier P, Riffo-Campos AL, Brebi P, Roa JC. Emerging role of miRNAs in the drug resistance of gastric cancer. Int J Mol Sci 2016; 17: 424.

9 Wang J, Yang M, Li Y, Han B. The role of microRNAs in the Chemoresistance of breast cancer. Drug Dev Res 2015; 76: 368-374.

10 Correia AL, Bissell MJ. The tumor microenvironment is a dominant force in multidrug resistance. Drug Resist Updat 2012; 15: 39-49.

11 Heidelberger C, Chaudhuri NK, Danneberg P, Mooren D, Griesbach L, Duschinsky $\mathrm{R}$ et al. Fluorinated pyrimidines, a new class of tumourinhibitory compounds. Nature 1957; 179: 663-666.

12 Bartel DP. microRNAs: genomics, biogenesis, mechanism, and function. Cell 2004; 116: 281-297.

13 Calin GA, Croce CM. microRNA signatures in human cancers. Nat Rev Cancer 2006; 6: 857-866.

14 Farazi TA, Hoell JI, Morozov P, Tuschl T. microRNAs in human cancer. Adv Exp Med Biol 2013; 774: 1-20.

15 Garofalo M, Croce CM. microRNAs: Master regulators as potential therapeutics in cancer. Annu Rev Pharmacol Toxicol 2011; 51: 25-43.

$16 \mathrm{He}$ L, Hannon GJ. microRNAs: small RNAs with a big role in gene regulation. Nat Rev Genet 2004; 5: 522-531.

17 Humphries B, Yang C. The microRNA-200 family: small molecules with novel roles in cancer development, progression and therapy. Oncotarget 2015; 6: 6472-6498.

18 Bracken CP, Khew-Goodall Y, Goodall GJ. Network-based approaches to understand the roles of miR-200 and other microRNAs in cancer. Cancer Res 2015; 75: 2594-2599.

19 Park SM, Gaur AB, Lengyel E, Peter ME. The miR-200 family determines the epithelial phenotype of cancer cells by targeting the E-cadherin repressors ZEB1 and ZEB2. Genes Dev 2008; 22: 894-907.

20 Lu Y, Lu J, Li X, Zhu H, Fan X, Zhu S et al. miR-200a inhibits epithelialmesenchymal transition of pancreatic cancer stem cell. BMC Cancer 2014; 14: 85.

21 Gregory PA, Bert AG, Paterson EL, Barry SC, Tsykin A, Farshid G et al. The miR-200 family and miR-205 regulate epithelial to mesenchymal transition by targeting ZEB1 and SIP1. Nat Cell Biol 2008; 10: 593-601.

22 Chen Y, Peng W, Lu Y, Chen J, Zhu YY, Xi T. miR-200a enhances the migrations of A549 and SK-MES-1 cells by regulating the expression of TSPAN1. J Biosci 2013; 38: 523-532.

23 Bracken CP, Li X, Wright JA, Lawrence DM, Pillman KA, Salmanidis M et al. Genome-wide identification of miR-200 targets reveals a regulatory network controlling cell invasion. EMBO J 2014; 33: 2040-2056.

24 Kang H, Kim C, Lee H, Kim W, Lee EK. Post-transcriptional controls by ribonucleoprotein complexes in the acquisition of drug resistance. Int J Mol Sci 2013; 14: 17204-17220.

25 Piya S, Kim JY, Bae J, Seol DW, Moon AR, Kim TH. DUSP6 is a novel transcriptional target of $\mathrm{p} 53$ and regulates p53-mediated apoptosis by modulating expression levels of $\mathrm{Bcl}-2$ family proteins. FEBS Lett 2012; 586: 4233-4240

26 Fulda S, Susin SA, Kroemer G, Debatin KM. Molecular ordering of apoptosis induced by anticancer drugs in neuroblastoma cells. Cancer Res 1998; 58: 4453-4460.
27 Agarwal V, Bell GW, Nam JW, Bartel DP. Predicting effective microRNA target sites in mammalian mRNAs. Elife 2015; 4: e05005.

28 Dweep H, Gretz N, Sticht C. miRWalk database for miRNA-target interactions. Methods Mol Biol 2014; 1182: 289-305.

$29 \mathrm{Mi} \mathrm{H}$, Thomas P. PANTHER pathway: an ontology-based pathway database coupled with data analysis tools. Methods Mol Biol 2009; 563: 123-140.

30 Thomas PD, Campbell MJ, Kejariwal A, Mi H, Karlak B, Daverman R et al. PANTHER: a library of protein families and subfamilies indexed by function. Genome Res 2003; 13: 2129-2141.

31 Wong N, Wang X. miRDB: an online resource for microRNA target prediction and functional annotations. Nucleic Acids Res 2015; 43: D146-D152.

32 Ellis LM, Hicklin DJ. Resistance to targeted therapies: refining anticancer therapy in the era of molecular oncology. Clin Cancer Res 2009; 15: 7471-7478.

33 Bracken CP, Gregory PA, Kolesnikoff N, Bert AG, Wang J, Shannon MF et al. A double-negative feedback loop between ZEB1-SIP1 and the microRNA-200 family regulates epithelial-mesenchymal transition. Cancer Res 2008; 68: 7846-7854.

34 Yu SJ, Hu JY, Kuang XY, Luo JM, Hou YF, Di GH et al. microRNA-200a promotes anoikis resistance and metastasis by targeting YAP1 in human breast cancer. Clin Cancer Res 2013; 19: 1389-1399.

35 Eades G, Yang M, Yao Y, Zhang Y, Zhou Q. miR-200a regulates Nrf2 activation by targeting Keap1 mRNA in breast cancer cells. J Biol Chem 2011; 286: 40725-40733.

36 Tsouko E, Wang J, Frigo DE, Aydogdu E, Williams C. miR-200a inhibits migration of triple-negative breast cancer cells through direct repression of the EPHA2 oncogene. Carcinogenesis 2015; 36: 1051-1060.

37 Eades G, Yao Y, Yang M, Zhang Y, Chumsri S, Zhou Q. miR-200a regulates SIRT1 expression and epithelial to mesenchymal transition (EMT)-like transformation in mammary epithelial cells. J Biol Chem 2011; 286: 25992-26002.

38 Mateescu B, Batista L, Cardon M, Gruosso T, de Feraudy Y, Mariani O et al. miR-141 and miR-200a act on ovarian tumorigenesis by controlling oxidative stress response. Nat Med 2011; 17: 1627-1635.

39 Su Y, He Q, Deng L, Wang J, Liu Q, Wang D et al. miR-200a impairs glioma cell growth, migration, and invasion by targeting SIM2-s. Neuroreport 2014; 25: 12-17.

40 Williams KC, Renthal NE, Condon JC, Gerard RD, Mendelson CR. microRNA-200a serves a key role in the decline of progesterone receptor function leading to term and preterm labor. Proc Natl Acad Sci USA 2012; 109: 7529-7534.

41 Keyse SM. Dual-specificity MAP kinase phosphatases (MKPs) and cancer. Cancer Metastasis Rev 2008; 27: 253-261.

42 Bermudez O, Pages G, Gimond C. The dual-specificity MAP kinase phosphatases: critical roles in development and cancer. Am J Physiol Cell Physiol 2010; 299: C189-C202.

43 Croonquist PA, Linden MA, Zhao F, Van Ness BG. Gene profiling of a myeloma cell line reveals similarities and unique signatures among IL-6 response, N-ras-activating mutations, and coculture with bone marrow stromal cells. Blood 2003; 102: 2581-2592.

44 Bloethner S, Chen B, Hemminki K, Muller-Berghaus J, Ugurel S, Schadendorf $D$ et al. Effect of common B-RAF and N-RAS mutations on global gene expression in melanoma cell lines. Carcinogenesis 2005; 26: 1224-1232.

45 Ramnarain DB, Park S, Lee DY, Hatanpaa KJ, Scoggin SO, Otu H et al. Differential gene expression analysis reveals generation of an autocrine loop by a mutant epidermal growth factor receptor in glioma cells. Cancer Res 2006; 66: 867-874.

46 Warmka JK, Mauro LJ, Wattenberg EV. Mitogen-activated protein kinase phosphatase-3 is a tumor promoter target in initiated cells that express oncogenic Ras. J Biol Chem 2004; 279: 33085-33092.

47 Xu S, Furukawa T, Kanai N, Sunamura M, Horii A. Abrogation of DUSP6 by hypermethylation in human pancreatic cancer. J Hum Genet 2005; 50: 159-167.

48 Okudela K, Yazawa T, Woo T, Sakaeda M, Ishii J, Mitsui H et al. Downregulation of DUSP6 expression in lung cancer: its mechanism and potential role in carcinogenesis. Am J Pathol 2009; 175: 867-881.

49 Chan DW, Liu VW, Tsao GS, Yao KM, Furukawa T, Chan KK et al. Loss of MKP3 mediated by oxidative stress enhances tumorigenicity and chemoresistance of ovarian cancer cells. Carcinogenesis 2008; 29: $1742-1750$ 
50 Furukawa T, Fujisaki R, Yoshida Y, Kanai N, Sunamura M, Abe T et al. Distinct progression pathways involving the dysfunction of DUSP6/MKP-3 in pancreatic intraepithelial neoplasia and intraductal papillary-mucinous neoplasms of the pancreas. Mod Pathol 2005; 18: 1034-1042.

51 Furukawa T, Sunamura M, Motoi F, Matsuno S, Horii A. Potential tumor suppressive pathway involving DUSP6/MKP-3 in pancreatic cancer. Am J Pathol 2003; 162: 1807-1815.

52 Smith TG, Karlsson M, Lunn JS, Eblaghie MC, Keenan ID, Farrell ER et al. Negative feedback predominates over cross-regulation to control ERK MAPK activity in response to FGF signalling in embryos. FEBS Lett 2006; 580: 4242-4245.

53 Tsang M, Maegawa S, Kiang A, Habas R, Weinberg E, Dawid IB. A role for MKP3 in axial patterning of the zebrafish embryo. Development 2004; 131: 2769-2779.

54 Marchetti S, Gimond C, Chambard JC, Touboul T, Roux D, Pouyssegur J et al. Extracellular signal-regulated kinases phosphorylate mitogenactivated protein kinase phosphatase 3/DUSP6 at serines 159 and 197, two sites critical for its proteasomal degradation. Mol Cell Biol 2005; 25: 854-864.

55 Bermudez O, Marchetti S, Pages G, Gimond C. Post-translational regulation of the ERK phosphatase DUSP6/MKP3 by the mTOR pathway. Oncogene 2008; 27: 3685-3691.

56 Kuwano Y, Kim HH, Abdelmohsen K, Pullmann R Jr, Martindale JL, Yang $\mathrm{X}$ et al. MKP-1 mRNA stabilization and translational control by RNA-binding proteins HuR and NF90. Mol Cell Biol 2008; 28: 4562-4575.

57 Li ZY, Hassan MQ, Jafferji M, Aqeilan RI, Garzon R, Croce CM et al. Biological functions of miR-29b contribute to positive regulation of osteoblast differentiation. J Biol Chem 2009; 284: 15676-15684.
58 Gu YF, Li DF, Luo QF, Wei CK, Song HM, Hua KY et al. Original article microRNA-145 inhibits human papillary cancer TPC1 cell proliferation by targeting DUSP6. Int J Clin Exp Med 2015; 8: 8590-8598.

59 Li G, Yu M, Lee WW, Tsang M, Krishnan E, Weyand CM et al. Decline in miR-181a expression with age impairs $T$ cell receptor sensitivity by increasing DUSP6 activity. Nat Med 2012; 18: 1518-1524.

60 Banzhaf-Strathmann J, Benito E, May S, Arzberger T, Tahirovic S, Kretzschmar $\mathrm{H}$ et al. microRNA-125b induces tau hyperphosphorylation and cognitive deficits in Alzheimer's disease. EMBO J 2014; 33: 1667-1680.

61 Becker LE, Takwi AA, Lu Z, Li Y. The role of miR-200a in mammalian epithelial cell transformation. Carcinogenesis 2015; 36: 2-12.

62 Liang $\mathrm{HH}$, Wei PL, Hung CS, Wu CT, Wang W, Huang MT et al. microRNA-200a/b influenced the therapeutic effects of curcumin in hepatocellular carcinoma (HCC) cells. Tumour Biol 2013; 34: 3209-3218.

This work is licensed under a Creative Commons Attribution-NonCommercial-NoDerivs 4.0 International License. The images or other third party material in this article are included in the article's Creative Commons license, unless indicated otherwise in the credit line; if the material is not included under the Creative Commons license, users will need to obtain permission from the license holder to reproduce the material. To view a copy of this license, visit http://creativecommons.org/licenses/by-nc-nd/4.0/

Supplementary Information accompanies the paper on Experimental \& Molecular Medicine website (http://www.nature.com/emm) 